Z Rheumatol 2020 $\cdot 79: 562-569$ https://doi.org/10.1007/s00393-020-00834-y Online publiziert: 10. Juli 2020

(c) Der/die Autor(en) 2020

J. Knitza ${ }^{1}$ J. Callhoff ${ }^{2}$ G. Chehab ${ }^{3} \cdot$ A. Hueber ${ }^{1,4} \cdot$ U. Kiltz ${ }^{5} \cdot$ A. Kleyer ${ }^{1} \cdot$ M. Krusche ${ }^{6}$. D. Simon ${ }^{1} \cdot$ C. Specker ${ }^{7} \cdot$ M. Schneider ${ }^{3}$. A. Voormann ${ }^{8} \cdot$ M. Welcker ${ }^{9} \cdot$ J. G. Richter ${ }^{3}$ ${ }^{1}$ Medizinische Klinik 3 - Rheumatologie und Immunologie, Universitätsklinikum Erlangen, FriedrichAlexander-Universität Erlangen-Nürnberg (FAU), Erlangen, Deutschland

${ }^{2}$ Programmbereich Epidemiologie, Deutsches Rheuma-Forschungszentrum (DRFZ), Berlin, Deutschland ${ }^{3}$ Poliklinik und Funktionsbereich Rheumatologie \& Hiller-Forschungszentrum Rheumatologie, Medizinische Fakultät, Universitätsklinikum Düsseldorf, Heinrich-Heine-Universität Düsseldorf, Düsseldorf, Deutschland

${ }^{4}$ Sektion Rheumatologie, Sozialstiftung Bamberg, Klinikum Bamberg, Bamberg, Deutschland

${ }^{5}$ Rheumazentrum Ruhrgebiet, Ruhr-Universität Bochum, Herne, Deutschland

${ }^{6}$ Medizinische Klinik mit Schwerpunkt Rheumatologie und Klinische Immunologie, Charité Universitätsmedizin, Berlin, Deutschland

${ }^{7}$ Klinik für Rheumatologie \& Klinische Immunologie, Evangelisches Krankenhaus, Kliniken Essen-Mitte, Essen, Deutschland

${ }^{8}$ Deutsche Gesellschaft für Rheumatologie e. V., Berlin, Deutschland

${ }^{9}$ Geschäftsführung, MVZ für Rheumatologie Dr. Martin Welcker GmbH \& RheumaDatenRhePort (rhadar), Planegg, Deutschland

\title{
Positionspapier der Kommission Digitale Rheumatologie der Deutschen Gesellschaft für Rheumatologie e. V.: Aufgaben, Ziele und Perspektiven für eine moderne Rheumatologie
}

sorgung zukommen zu lassen und den Transfer aktueller wissenschaftlicher Erkenntnisse aus der Forschung in die Praxis zu vollziehen. Ein Anliegen, dem sich die DGRh stellen muss, ist die Digitalisierung von Leitlinien. Die Kommission begleitet deshalb die Entwicklung und Auswahl geeigneter informationstechnologischer (IT) Applikationen.

Ein ebenso gefragtes Instrument in der Betreuung rheumatologischer Patienten sind die sog. „Therapieüberwachungsbögen " [2] der DGRh. An die Kommission wurde von der Projektgruppe der Arbeitsgemeinschaft Regionaler Kooperativer Rheumazentren (AGRZ) der DGRh die Aufgabe herangetragen, die Entwicklung eines Datenbankmodells und die Auswahl einer IT-Anwendung zu begleiten, das die komfortable, fortlaufende Aktualisierung dieser Bögen ermöglicht und unter anderem durch Prozessopti- mierung für eine zusätzliche Professionalisierung dieses wichtigen Angebotes sorgt.

Interoperabilität, Nutzen und Sicherheit sind gleichermaßen für Anbieter und Anwender im eHealth-Bereich maßgebliche Kriterien, an denen sich digitale Produkte messen lassen müssen. DerEinsatz von IT-Plattformen etwa ist angesichts stetig wachsender Datenmengen, zunehmender Komplexität und allen damit verbundenen Chancen und Risiken eine enorme Aufgabe für unser Gesundheitswesen. Dies betrifft z. B. die Erfassung und Auswertung von Patientendaten in Registern und Kohortenstudien.

Die DGRh bringt sich deshalb mit Blick auf ihr Ziel, Forschung in der Rheumatologie zu fördern, aktiv in die Suche nach geeigneten Anwendungen, Applikationen (Apps), Datenbanken, Electronic-Data-Capture(EDC)-Syste- 
Tab. 1 Von Rheumatologen beim DGRh(Deutsche Gesellschaft für Rheumatologie e. V.)-Kongress 2018 empfohlene Apps für Kollegen und Patienten [4]

\begin{tabular}{|c|c|c|c|c|c|c|}
\hline App Name & Zweck & $\begin{array}{l}\text { Anteil an App-Empfehlun- } \\
\text { gen für Kollegen } n=52 \text {, } \\
n(\%)\end{array}$ & $\begin{array}{l}\text { Anteil an App-Empfehlun- } \\
\text { gen für Patienten } n=8, \\
n(\%)\end{array}$ & Preis & iOS & Android \\
\hline Labcal & Berechnungstool & $1(2)$ & $x$ & Kostenlos & $\mathrm{Ja}$ & Nein \\
\hline Medcalx & Berechnungstool & $4(8)$ & $x$ & Kostenlos & $\mathrm{Ja}$ & Nein \\
\hline $\begin{array}{l}\text { PAH - Woche für } \\
\text { Woche }\end{array}$ & Berechnungstool & $1(2)$ & $x$ & Kostenlos & Ja & Ja \\
\hline $\begin{array}{l}\text { Calculate by } \\
\text { QXMD }\end{array}$ & Berechnungstool & $4(8)$ & $x$ & Kostenlos & $\mathrm{Ja}$ & Ja \\
\hline Rheuma helper & Berechnungstool & $7(13)$ & $1(13)$ & Kostenlos & $\mathrm{Ja}$ & $\mathrm{Ja}$ \\
\hline Ada & Diagnoseunterstützung & $1(2)$ & $1(13)$ & Kostenlos & Ja & Ja \\
\hline Isabel & Diagnoseunterstützung & $1(2)$ & $x$ & Kostenpflichtig & Nein & Nein \\
\hline AmiKo Desitin & Medikamenteninformation & $1(2)$ & $x$ & Kostenlos & $\mathrm{Ja}$ & $\mathrm{Ja}$ \\
\hline Arznei aktuell & Medikamenteninformation & $11(21)$ & $1(13)$ & Kostenlos & $\mathrm{Ja}$ & $\mathrm{Ja}$ \\
\hline $\begin{array}{l}\text { Arzneimittel } \\
\text { Pocket }\end{array}$ & Medikamenteninformation & $2(4)$ & $x$ & Kostenlos & $\mathrm{Ja}$ & Ja \\
\hline Corticonverter & Medikamenteninformation & $1(2)$ & $x$ & Kostenlos & Nein & Nein \\
\hline EKO2go & Medikamenteninformation & $1(2)$ & $x$ & Kostenlos & $\mathrm{Ja}$ & Ja \\
\hline Embryotox & Medikamenteninformation & $2(4)$ & $3(38)$ & Kostenlos & $\mathrm{Ja}$ & Ja \\
\hline Pneumotox & Medikamenteninformation & $1(2)$ & $x$ & Kostenlos & Nein & Nein \\
\hline Amboss Wissen & Nachschlagewerk & $4(8)$ & $x$ & Kostenpflichtig & $\mathrm{Ja}$ & $\mathrm{Ja}$ \\
\hline $\begin{array}{l}\text { DocCheck Flexi- } \\
\text { con }\end{array}$ & Nachschlagewerk & $2(4)$ & $x$ & Kostenlos & $\mathrm{Ja}$ & Ja \\
\hline KBV2GO! & Nachschlagewerk & $1(2)$ & $x$ & Kostenlos & $\mathrm{Ja}$ & $\mathrm{Ja}$ \\
\hline Medscape & Nachschlagewerk & $1(2)$ & $x$ & Kostenlos & $\mathrm{Ja}$ & Ja \\
\hline UpToDate & Nachschlagewerk & $4(8)$ & $x$ & Kostenlos & $\mathrm{Ja}$ & $\mathrm{Ja}$ \\
\hline RheumaLive & Symptom-Tracking & $2(4)$ & $2(25)$ & Kostenlos & Ja & Ja \\
\hline
\end{tabular}

men für klinische Studien, Produkten für eLearning und ePublishing ein. Sie beteiligt sich zudem am Aufbau geeigneter Plattformen. Digitale Medizin wird dabei zum Gegenstand der Versorgungsforschung. Hierbei sind Agilität, Effizienz und abermals Datensicherheit und -schutz nur einige der Kriterien, an denen sich die Beteiligten orientieren müssen. Der Kommission kommt eine wichtige Rolle in der Beratung der DGRh bei diesen weitreichenden Entscheidungen zu. Eine Aufgabe umfasste bereits die Entwicklung eines Anforderungskatalogs für eine IT-Plattform Rheumatologie. Nur ein Aspekt ist dabei die Beachtung moderner (IT-)Standards wie etwa der FHIR(Fast Healthcare Interoperability Resources)-Standard, der den modernen Datenaustausch zwischen Softwaresystemen im Gesundheitswesen unterstützt, und „FAIR (Findable, Accessible, Interoperable, Reusable) Data“, einem Prinzip, das ein nachhaltiges For- schungsdatenmanagement sichern soll [3].

Zu den Aufgaben der Kommission gehört darüber hinaus die - möglichst fortlaufende - Evaluation von Gesundheitsapplikationen (Apps und andere). Deren unten beschriebene Bedeutung im Medizin- und Gesundheitsbereich im Allgemeinen wächst ebenso zügig wie in der Rheumatologie im Besonderen. Konkret fällt der Kommission etwa die Definition von Produktvoraussetzungen von Applikationen $\mathrm{zu}$, in die sie im nationalen Kontext eingebunden ist. In dem Zusammenhang ist sie über einzelne Kommissionsmitglieder auch in Projekte der Deutschen Gesellschaft für Innere Medizin e. V. (DGIM) eingebunden.

Die DGRh nimmt in hohem Maße ihren Auftrag der wissenschaftlichen Fortbildung von Rheumatologen wahr. Hierbei spielen digitale Angebote eine immer größere Rolle. Die Kommission Digitale Rheumatologie der
DGRh unterstützt die Fachgesellschaft und damit auch die Rheumatologische Fortbildungsakademie bei der Sichtung vorhandener e-Learning-Projekte und deren Evaluation. Sie will sich dabei auch Zielgruppenbefragungen bedienen, um Angebote möglichst nutzerorientiert gestalten zu können.

Weitere zukunftsweisende Themen, mit denen sich die Kommission befasst, sind „künstliche Intelligenz" und „machine learning“, Telemedizin und Digital Devices (dazu zählen z. B. tragbare Sensoren wie Aktivitätstracker). Die Kommission plant zu diesen Themen jährlich eine Sitzung im Rahmen des Kongresses der DGRh. Dabei bezieht sie Rheumatologen und Vertreter der Fachgesellschaft ebenso ein wie Experten, die Impulse aus anderen Fachgebieten einbringen können - Juristen, IT-Experten und Anbieter digitaler Produkte. Die Arbeit der Kommission erfolgt in enger Zusammenarbeit mit anderen Kommissionen 
(z. B. Kommission Leitlinien, Kommission Pharmakotherapie, Kommission für studentische Ausbildung), um Redundanzen zu vermeiden und synergistische Effekte zu nutzen.

$\mathrm{Zu}$ beachten ist bei der Auseinandersetzung mit den Themen der medizinischen und rheumatologischen Digitalisierung auch die angemessene Koordination und Kooperation. Dies gilt sowohl unter Rheumatologen als auch fach- und sektorübergreifend, interprofessionell und international etwa mit der europäischen Dachorganisation European League Against Rheumatism (EULAR). Auch hierbei kommt der Kommission Digitale Rheumatologie in enger Abstimmung mit der DGRh eine wichtige Aufgabe zu. Angesichts der großen Dynamik des Gebietes und aufgrund der aktuell sehr zahlreichen Aktivitäten u. a. des Bundesministeriums für Gesundheit und weiterer politischer Stakeholder ist die Kommission häufig gefragt, wenn es darum geht, Stellungnahmen abzugeben. Sämtliche Verlautbarungen, Publikationen und Positionierungen erfolgen in Rücksprache mit dem Vorstand der DGRh, der diese verantwortet.

Die Kommission hofft, dass die digitale Rheumatologie die Versorgung der Patienten z.B. durch eine effizientere Nutzung knapper personeller Ressourcen optimieren wird. Digitale Medizin soll dabei in den nächsten Jahren eine zunehmend personalisierte, flexible und präventive Behandlung ermöglichen. Die bessere Verfügbarkeit und Aufbereitung wertvoller klinischer Daten soll die evidenzbasierte Therapie nachhaltig unterstützen. Therapien sollen durch niedrigschwellige Zugangsmöglichkeiten schneller etabliert und mittels digitalen Monitorings deren Wirksamkeit und Sicherheit umfassender beurteilt werden. Langjährig und fachübergreifend wird in der Digitalisierung das Thema EDV-gestützte Überwachung von Arzneimittelinteraktionen thematisch bearbeitet, ohne dass sich dazu ein Konzept nachhaltig durchgesetzt hat. Entsprechende Entwicklungen und Lösungskonzepte werden von Mitgliedern der Kommission beobachtet.

Nachfolgend sind bereits angesprochene und weitere Themengebiete, mit

Z Rheumatol 2020 · 79:562-569 https://doi.org/10.1007/s00393-020-00834-y

(c) Der/die Autor(en) 2020

J. Knitza · J. Callhoff · G. Chehab · A. Hueber · U. Kiltz · A. Kleyer · M. Krusche · D. Simon • C. Specker $\cdot$ M. Schneider $\cdot$ A. Voormann $\cdot$ M. Welcker $\cdot$ J. G. Richter

Positionspapier der Kommission Digitale Rheumatologie der Deutschen Gesellschaft für Rheumatologie e. V.: Aufgaben, Ziele und Perspektiven für eine moderne Rheumatologie

\section{Zusammenfassung}

Die Digitalisierung im Gesundheitswesen ist für die Rheumatologie eine ebenso große Herausforderung wie für andere medizinische Fachgebiete. Die Deutsche Gesellschaft für Rheumatologie e. V. (DGRh) will diesen Prozess aktiv gestalten und davon profitieren. Mit der Gründung der Kommission Digitale Rheumatologie hat sie ein Gremium geschaffen, das die damit verbundenen Aufgaben bearbeitet, die DGRh zu Fragestellungen berät und sich positioniert. Für die DGRh berührt dies verschiedenste Bereiche der Digitalisierung in Medizin und Rheumatologie. Dieses Positionspapier legt die aktuell von der Kommission bearbeiteten Themengebiete, Entwicklungen und identifizierten Aufgaben dar.

Schlüsselwörter

eHealth · mHealth · Digitalisierung .

Versorgungsforschung $\cdot$ App

\section{Position paper of the commission on digital rheumatology of the German Society of Rheumatology: tasks, targets and perspectives for a modern rheumatology}

\section{Abstract}

Digitalization in the healthcare system is a great challenge for rheumatology as for other medical disciplines. The German Society for Rheumatology (DGRh) wants to actively participate in this process and benefit from it. By founding the commission on digital rheumatology, the DGRh has created a committee that deals with the associated tasks, advises the DGRh on questions and positions associated with digital health.
For the DGRh, this affects the most diverse areas of digitalization in medicine and rheumatology. This position paper presents the topics and developments currently handled by the commission and the tasks identified.

Keywords

eHealth · mHealth · Digitalization · Health services research $\cdot$ App denen die Kommission sich beschäftigt, weiter ausgeführt.

\section{Apps in der Rheumatologie}

Eine Umfrage der Arbeitsgemeinschaft Junge Rheumatologie (AGJR) DGRh konnte zeigen, dass die Nutzung medizinischer Apps in der rheumatologischen Routineversorgung in den letzten Jahren zunahm [4]. Im Jahr 2018 nutzte bereits nahezu die Hälfte der befragten Rheumatologen (49\%) entsprechende Apps. Insgesamt identifizierte die Umfrage 20 empfehlenswerte Apps (•Tab. 1), unter denen jedoch nur 2 rheumaspezifische Apps waren. In einer zweiten Arbeit [5] der AGJR wurden deutsche rheumaspezifische Apps systematisch identifiziert und anhand der validierten MobileApp-Rating-Scale (MARS) [6] bewertet. Sechzehn Apps erfüllten die Einschlusskriterien. Sieben Apps richteten sich an Ärzte, 9 an Patienten. Am besten bewertet wurde die App der Deutschen RheumaLiga: „Rheuma-Auszeit“. Die Qualität der Apps war sehr heterogen, und es konnten keine Wirksamkeitsstudien der Apps identifiziert werden. Im Rahmen des DGRh-Kongresses 2020 werden die Ergebnisse der Anwendung der „Semiautomated Retrospective App Store Analysis"-Methode auf den Apple App Store, zur Identifizierung relevanter, qualifizierter deutschsprachiger Apps für das Fachgebiet Rheumatologie präsentiert. Eine systematische Literaturrecherche [7] belegte nur für wenige identifizierte 
Studien eine Outcome-Verbesserung. Außerdem waren viele der in Studien evaluierten Apps nicht mehr kommerziell erhältlich. Analog zur EULAR (European League Against Rheumatism) [8] konnten in der Publikation entsprechende Empfehlungen für AppHersteller [5] ermittelt werden. Besonders betont wurde die Zusammenarbeit mit Patienten bei der Entwicklung von Apps. Entgegen dem Digitalen-Versorgung-Gesetz (DVG) soll laut Punkt 10 der EULAR-Empfehlungen vor einer Implementierung bzw. Förderung entsprechender Apps zunächst eine KostenNutzen-Analyse durchgeführt werden. Eine Patientenumfrage [9] zeigte, dass die große Mehrheit aller Patienten (96\%) unter Berücksichtigung des geltenden Datenschutzes bereit ist, App-Daten auch für Forschungszwecke zur Verfügung zu stellen. Die Arbeit beschreibt außerdem, dass Patienten sich von einer App neben weiteren Inhalten insbesondere Informationen zur Erkrankung, zu Medikamenten und zur Ernährung wünschen. Die für das Register „Rheuma, Kinderwunsch und Schwangerschaft“ (Rhekiss) entwickelte responsive App wird beispielsweise sehr gut von den Patientinnen angenommen, um die entsprechenden patientenberichteten Daten zu dokumentieren [10]. Durch das DVG haben Patienten nun einen Anspruch auf digitale Gesundheitsanwendungen (DiGA) inklusive Gesundheits-Apps, die Medizinprodukte darstellen und entsprechend dem Bundesinstitut für Arzneimittel und Medizinprodukte (BfArM) als verordnungsfähig eingestuft wurden sowie im entsprechenden DiGA-Verzeichnis gelistet werden. Die Kommission schließt sich den EULAR- [8] und AGJR- [5] Empfehlungen an und möchte entsprechende Wirksamkeitsstudien nach Möglichkeit aktiv unterstützen.

\section{Digitale Diagnose- beschleunigung}

Eine der größten Herausforderungen der Rheumatologie ist nach wie vor die schnelle Diagnosestellung [11, 12], die bei der rheumatoiden Arthritis (RA) auch für ein gutes Therapieansprechen entscheidend ist [13]. Aktuell können
Rheumatologen die große Zahl an Erstvorstellungsanfragen nur begrenzt zügig bearbeiten. Eine Triage ist unabdingbar, und es existieren verschiedenste lokale Ansätze [14]. Neunzig Prozent der rheumatologischen Patienten nutzen regelmäßig ein Smartphone [9]. Wie bereits vor ca. 15 Jahren sucht fast die Hälfte der Patienten im Internet (vor dem Arzttermin) medizinische Informationen (u.a. $\mathrm{zu}$ bestehenden Symptomen) [15, 16]. Aktuell liegt bei bis zu $60 \%$ der Erstvorstellungen keine entzündlich rheumatische Grunderkrankung vor [17], und lange Wartezeiten sind die Norm. Kostenintensive analoge Optimierungsmöglichkeiten - wie die Terminservicestellen - haben bisher nicht zum gewünschten Erfolg geführt [18]. Niedrigschwellige, skalierbare und kostenarme digitale Diagnoseunterstützungssysteme („diagnostic decision support system“ [DDSS]) werden aktuell ausgiebig entwickelt. Grob unterscheiden kann man Systeme, die sich an Patienten oder Ärzte richten, fachübergreifende/fachspezifische, diagnosespezifische Systeme und Systeme, die konkrete Verdachtsdiagnosen mit Wahrscheinlichkeiten angeben. Joint-Pain-Assessment-Tool (JPAST) (https://eithealth.eu/project/jpast/) ist ein rheumaspezifischer europäischer Algorithmus, der aktuell an mehreren Unikliniken validiert wird [19]. Rheport (www.rheport.de) und Rheuma-VOR [20] (www.rheuma-vor.de) sind ebenfalls rheumaspezifische Tools, die bereits in einigen Bundesländern zur Terminvergabe verwendet werden. Ada (www. ada.com/de/) ist ein fachübergreifender „Symptom-Checker“, der konkrete Verdachtsdiagnosen und Handlungsempfehlungen erstellt. Aktuell läuft die Rekrutierung einer prospektiven multizentrischen Studie, die Ada mit Rheport vergleicht (DRKS Studienidentifier DRKS00017642). Ein diagnosespezifisches (axiale Spondylarthritis), an der Charité - Universitätsmedizin Berlin entwickeltes Tool findet sich auf www. bechterew-check.de. Durch ein Update konnte hier zuletzt die Sensitivität zur Detektion einer axialen Spondylarthritis auf $90 \%$ erhöht werden [21]. Seit 2009 steht der vom Universitätsklinikum Düsseldorf/Rheumazentrum Rhein-Ruhr e. V. validierte RheumaCheck online zur Verfügung (https://rheuma-check.de/) und wird von ca. 4500 Usern/Monat genutzt [22]. In Spanien schloss sich die Gesellschaft für Rheumatologie mit Hausärzten zusammen und entwickelte gemeinsam eine App zur Optimierung der Überweisung von Patienten [23]. Die digitale Dokumentation der Symptome und die digitale Terminvergabe schonen im besten Fall Ressourcen und schaffen passiv einen wertvollen $\mathrm{Da}$ tensatz für die Versorgungsforschung. Entwicklungen zu dieser Thematik wird die Kommission Digitale Rheumatologie weiter regelmäßig begleiten.

\section{Digitale Patient-reported- outcome-Instrumente}

Patient-reported-outcome(PRO)-Instrumente bieten die Möglichkeit, Gesundheitszustände (z. B. Lebensqualität, Fatigue), die nur direkt durch den $\mathrm{Pa}$ tienten beurteilt und erhoben werden können, standardisiert zu erfassen. PRO werden zur Therapieerfolgsbeurteilung in klinischen Studien gefordert, finden aber auch zunehmende Anwendung in der klinischen Routine. In Zeiten knapper Ressourcen, Tight-control-Konzepten und "Shared Decision Making" scheinen sie die Arzt-Patienten-Interaktion und das Patientenmanagement sinnvoll unterstützen zu können [24]. Ihre Nutzung hat nachweislich zu einer Verbesserung des Outcomes geführt [25]. Langfristig sind durch die Implementierung von PROs in der Routine positive Effekte auf die Kosten des Gesundheitswesens anzunehmen.

Der Einsatz elektronischer PRO (ePRO) kann die Nutzung von PROs in der täglichen klinischen Praxis der Rheumatologie unterstützen [26]. Wesentliche Vorteile elektronischer Gesundheitsakten und anderer Systeme, die ePROs inkludieren, stellen dabei das automatisierte Scoring sowie die langfristige systematische patientenzentrierte Datenerfassung in strukturierter und standardisierter Form dar [27]. Sie gewährleisten eine Darstellung der Scores im Krankheitsverlauf, um Therapieentscheidungen $\mathrm{zu}$ unterstützen und Therapieerfolge $\mathrm{zu}$ beurteilen. ePRO- 
Daten aus elektronischen Gesundheitsakten werden - auch aufgrund des neuen Digitalen-Versorgung-Gesetzes - zunehmend in patientenzentrierte Forschung, Register und andere Sekundärdatennutzungen einfließen können und so die Möglichkeit bieten, den Wissens- und Forschungsstand $\mathrm{zu}$ verbessern sowie neue Prozessabläufe in der rheumatologischen Versorgung zu schaffen [27].

Für die Routineversorgung stehen verschiedene computergestützte Datenerfassungstools, Websites und mobile Applikation zur Verfügung, mit denen PROs gesammelt werden können und die das (Selbst-)Monitoring bereits abseits ambulanter Routinevorstellungen unterstützen [24]. Evaluationen moderner, mobiler Applikationen, die Aspekte von Remote-Monitoring u.a. durch Nutzung von ePROs beinhalten, sind Bestandteile aktueller rheumatologischer Forschungsprojekte [5, 9, 28, 29]. Wirksamkeitsnachweise für mobile Applikationen für die rheumatologische Versorgung sind bislang rar.

Die elektronische Erfassung von Daten klinischer Studien einschließlich ePRO-Systemen soll im Vergleich zu papierbasierten Methoden zu einer genaueren und vollständigeren Datenerfassung, einer verbesserten Compliance und einer besseren Patientenakzeptanz führen können [30]. Aktuell nutzen ca. $25 \%$ der deutschen Rheumatologen routinemäßig ePROs [31]. In einer Studie bei Patienten mit systemischem Lupus erythematodes (SLE) konnte bereits eine signifikant höhere Therapieadhärenz bei Nutzung von ePRO gezeigt werden [32]. Es wird zudem antizipiert, dass elektronische Tagebücher den Recall Bias reduzieren und die Qualität der PRODatenerhebung verbessern [30].

Mit dem Aufbau des oben genannten „Verzeichnis der Digitalen Gesundheitsanwendungen“" (DiGA-Verzeichnis) durch das BfArM wird ein Verzeichnis etabliert, das die digitalen Gesundheitsanwendungen aufführen wird, die den im Gesetz festgelegten Anforderungen entsprechen und somit erstattungsfähig sind. Welche ePRO-Applikationen dort zu finden sein werden, bleibt zum gegenwärtigen Zeitpunkt offen. Nach aktuel- lem Stand haben 2 Anbieter Bestrebungen, dort gelistet $\mathrm{zu}$ werden.

Die Kommission Digitale Rheumatologie hält die Bearbeitung des Themengebietes elektronischer Patient-reported-outcome-Instrumente für relevant, um Behandlungskapazitäten in der klinischen Routine zukünftig sinnvoller nutzen zu können. So könnten ePROs auch zur Triage herangezogen werden.

\section{Digitale Lehranwendungen}

Die studentische Ausbildung im Fach Rheumatologie ist, wie eine Evaluation der DGRh aufgedeckte, verbesserungswürdig. So kommen im Median Studierende während ihrer gesamten Universitätsausbildung mit nur 5 Patienten mit rheumatischen Erkrankung in Kontakt und nehmen im Median an nur $6 \mathrm{~h}$ praktischer Übung teil [33].

Um nachhaltige Qualitätsverbesserungen in der Ausbildung zu erreichen und das Fach Rheumatologie für Studierende sichtbar zu machen, sind deshalb rheumadidaktische Lehrkonzepte mit digitalen und virtuellen Elementen in der curricularen rheumatologischen Lehre notwendig. Neue Visualisierungstechniken in Kombination mit sinnvollen medizindidaktischen Ansätzen könnten die rheumatologische Lehre optimieren und das studentische Interesse am Fachgebiet Rheumatologie wecken respektive erhöhen.

Bereits heute können neuartige Darstellungsformen von Gelenkpathologien mittels virtueller Realität (VR) die konventionelle Lehre ergänzen. Eine dieser VR-Applikationen (Rheumalityœ, Eli Lilly, USA), eine Software, die nur zugangsgeschützt verfügbar ist, wurde an der Friedrich-Alexander-Universität Erlangen-Nürnberg entwickelt und wird an mehreren medizinischen Fakultäten zur Lehre aktuell und zukünftig eingesetzt (Jena, Magdeburg, Mannheim, Gießen, Düsseldorf, Berlin). Rheumality® erlaubt, die Darstellung von Knochenstrukturen realer Patienten mit rheumatischen Erkrankungen in einer virtuellen Welt. Studierende können in die pathologisch veränderten Knochen dieser Patienten eintauchen und sie durch „Erleben“ (Immersion) nachhal- tig verstehen. Die VR-Applikation wird fortlaufend weiterentwickelt, um das Spektrum rheumatischer Erkrankungen möglichst vollständig abzubilden [34].

Neue digitale Ansätze können das Erlernen muskuloskeletaler Bildgebung erleichtern. So bieten digitale Lernmodule für den muskuloskeletalen Ultraschall eine didaktisch wertvolle Unterstützung der Präsenzlehre im Rahmen von Arthrosonographiekursen für Studierende [35]. Diese digitalen Lernmodule ermöglichen die Vor- und Nachbereitung von Untersuchungsübungen und bieten während der Übungen konkrete Orientierungshilfen. Zudem erlauben die Lernmodule den Studierenden das anschauliche Nachvollziehen der Untersuchungsabläufe verschiedener Gelenke. Für die Etablierung der Online-Lernmodule wurden die sonographischen Untersuchungsabläufe mithilfe zahlreicher Beispielbilder abgebildet und Standardschnitte mit ergänzenden Anatomieskizzen zur Orientierung erstellt. Ergänzend dazu wurden pathologische Befunde bereitgestellt, die für jedes Gelenk einen exemplarischen Überblick über die häufigsten Pathologien geben. Momentan werden die Lerninhalte auf der Plattform www.sonographie.org implementiert. Diese digitalen Module sind mittelfristig frei zugänglich und stehen Interessierten niedrigschwellig zur Verfügung. Dadurch sollen neue digitale Lehransätze auch an anderen Institutionen unkompliziert eingesetzt werden können. Weitere Tätigkeiten in diesem Bereich werden von der Kommission Digitale Rheumatologie aktiv begleitet.

\section{Regularien}

Hohe Aufmerksamkeit hat das vom Deutschen Bundestag am 07.11.2019 beschlossene, am 18.12.2019 im Bundesgesetzblatt veröffentlichte und am 01.01.2020 in Kraft getretene DigitaleVersorgungs-Gesetz erlangt [36]. Es soll dabei helfen, den Digitalisierungsprozess im deutschen Gesundheitswesen weiter voranzutreiben und die Innovationskraft des Gesundheitssystems zu fördern [36].

Das Gesetz stellt alle Stakeholder des Gesundheitswesens vor große Herausforderungen, da „ein Leistungsanspruch 
der Versicherten auf digitale Gesundheitsanwendungen (,DiGA ${ }^{c}$ ) geschaffen und ein Verfahren beim Bundesinstitut für Arzneimittel und Medizinprodukte (BfArM) etabliert wird, mit dem über die Leistungserbringung in der Regelversorgung entschieden wird“ [36]. Digitale Gesundheitsanwendungen sind dabei Medizinprodukte, die nach der neuen, 2021 wirksam werdenden EU-Medizinprodukte-Verordnung (,Medical Device Regulation") einer niedrigen Risikoklasse (Klasse I: „nichtinvasive Produkte“, oder IIa: „Software, die dazu bestimmt ist, Informationen zu liefern, die zu Entscheidungen für diagnostische oder therapeutische Zwecke herangezogen werden") zuzuordnen sind.

Neben diesen als „Gesundheits-Apps auf Rezept" bekannt gewordenen DiGAs wird der Einsatz von Telemedizin (Videosprechstunde) gefördert. Die Erweiterung der Telematikinfrastrukturim Gesundheitswesen (TI) wird vorangetrieben (z.B. Krankenhäuser und Apotheken). Verwaltungsprozesse sollen vereinfacht werden (z. B. elektronische Heilund Hilfsmittelverordnung).

Das DVG sieht darüber hinaus die Speicherung der Gesundheitsdaten in elektronischen Patientenakten (ePA) vor, die Krankenkassen ihren Versicherten spätestens ab 01.01.2021 anbieten müssen. Daten für die Versorgungsforschung sollen schneller verfügbar sein. Die Krankenkassen sollen ihre Abrechnungsdaten pseudonymisiert an den GKV(gesetzliche Krankenversicherung)-Spitzenverband liefern, von dort sollen sie zunächst an das Forschungsdatenzentrum beim BfArM weitergeleitet werden. Nach Anonymisierung und Aggregierung am Forschungsdatenzentrum stehen sie für gesetzlich definierte Forschungseinrichtungen (Hochschulen, Universitätskliniken und Institute, wie z.B. das IQWiG [Institut für Qualität und Wirtschaftlichkeit im Gesundheitswesen]) zur Verfügung. Relevante datenschutzrechtliche Aspekte, die die Vorgaben der Datenschutz-Grundverordnung umfassend berücksichtigen, sind bei diesen Entwicklungen zu berücksichtigen und waren mehrfach Gegenstand der Diskussion um das Gesetz. Die erste Rechtsverordnung zum DVG wurde am
20.04.2020 bereits im Bundesanzeiger veröffentlicht [37].

Die Umsetzung und Auslegung des Gesetzes wird Auswirkungen auf die rheumatologische Versorgung haben. Die weiteren Entwicklungen werden von der Kommission Digitale Rheumatologie beobachtet, Konsequenzen für die DGRh aufgezeigt. Jüngst wurde von der DGRh und der Kommission eine Stellungnahme zu dem Referentenentwurf „Verordnung über das Verfahren und die Anforderungen der Prüfung der Erstattungsfähigkeit digitaler Gesundheitsanwendungen in der gesetzlichen Krankenversicherung" (DigitaleGesundheitsanwendungen-Verordnung - DiGAV) des Bundesministeriums für Gesundheit und eine weitere $\mathrm{zu}$ dem Entwurf der oben genannten Rechtsverordnung zum DVG abgegeben [38].

\section{Dokumentationsplattform RheMIT}

Die Erhebung homogener, transparenter Versorgungsdaten ist ein gemeinsames Anliegen vieler Parteien in der rheumatologischen Versorgungslandschaft. In der Vergangenheit wurden dafür unterschiedliche Softwarelösungen durch oder in Kooperation mit den Parteien eingesetzt (z.B. DocuMed.rh, RheumDok). Der Berufsverband Deutscher Rheumatologen e.V. (BDRh) betreibt in Zusammenarbeit mit einem Softwarehersteller die Bereitstellung einer Software-basierten modernen Dokumentationsplattform, um die bisherige Softwarelösung RheumaDok abzulösen. In Kooperation mit der DGRh, dem Deutschen Rheuma-Forschungszentrum Berlin und dem Verband der Rheumatologischen Akutkliniken e.V. soll eine zeitgemäße Dokumentationsplattform bereitgestellt werden, die perspektivisch alle Projekte der Parteien unterstützt, welche die Erhebung von Versorgungs- und Forschungsdaten vorsehen. Die Dokumentationsplattform wird in mindestens 2 Varianten bereitgestellt. Zum einen wird sie unter dem Namen RheMIT mit einer Basisausführung für den Einsatz in der Versorgungsforschung und anderen Projekten zur Verfügung gestellt. Zum anderen soll die
Dokumentationsplattform unter dem Namen RheMITplus erweiterte Funktionen zum Patientenmanagement bieten. Perspektivisch sind Weiterentwicklungen der Plattform unter Beachtung der Europäischen Medizinprodukteverordnung (MDR) möglich.

\section{Digitale Entwicklungen im Kontext Leitlinien}

Die Entwicklung von (interdisziplinären) Leitlinien bietet sich aufgrund ihrer Komplexität, den relativen starren Entwicklungsschritten und der Nutzung großer Datenmengen gerade für die Verwendung digitaler Methoden an. Schon bisher wurden von der DGRh partiell digitale Applikationen wie "Ryyan“ zur Abstractselektion im Rahmen einer systematischen Literatursuche oder die Technologie- und Methodenplattform für die vernetzte medizinische Forschung e.V. (TMF) zur Erstellung der Langfassung einer Leitlinie genutzt (https://www.tmf-ev.de). Es fehlen bisher allerdings digitale Anwendungen, die in den verschiedenen Entwicklungsschritten zuverlässig und praktikabel die verschiedenen Anforderungen erfüllen konnten.

Neben der Optimierung der Erstellung von Leitlinien durch digitale Systeme muss im Rahmen der Leitlinienarbeit klar gefordert werden, dass publizierte Leitlinien in einer digital aufbereiteten Form dem Anwender zur Verfügung stehen. Bei dieser Aufbereitung sind der unterschiedliche Wissensstand (Studierende bis Facharzt) und die divergierenden Interessenlagen zu berücksichtigen. Die DGRh kooperiert hier bereits mit der Arbeitsgemeinschaft der Wissenschaftlichen Medizinischen Fachgesellschaften e.V. (AWMF), die im Rahmen eines BMBF(Bundesministerium für Bildung und Forschung)-geförderten Projektes ein Pilotprojekt zur Leitliniendigitalisierung mit dem Namen DissoLVe (Digitalisierung von Leitlinienwissen zur Überwindung von Grenzen des Medizinischen Wissensmanagements: Modellprojekt für die Entwicklung hochwertiger Leitlinien und deren Verbreitung über Apps) gestartet hat (Auftaktworkshop Oktober 2019). Die AWMF plant in 
diesem Projekt perspektivisch alle Schritte im „Lebenszyklus“ von Leitlinien digital zu unterstützen und dadurch v. a. die Leitlinienarbeit für Autoren und Koordinatoren zu erleichtern.

Insbesondere die inhaltliche Bewertung der Evidenz stellt eine große Herausforderung dar, und die AWMF möchte hier mit dem Tool MAGIC (Making GRADE the Irresistible Choice) eine Arbeitserleichterung herstellen. Allerdings setzt die Applikation ein hohes methodisches Wissen voraus, da die Software GRADE Bewertung operationalisiert. Zukünftig sind neben der digitalen Erstellung der Leitlinien auch die Implementierung der Leitlinien in die klinische Anwendbarkeit via digitale Applikationen notwendig, um einen niedrigschwelligen Ansatz bieten zu können und dem veränderten Wissenstransfer Rechnung zu tragen.

\section{Fazit}

Dieses Positionspapier legt die aktuell von der Kommission Digitale Rheumatologie bearbeiteten Themengebiete, Entwicklungen und identifizierten Aufgaben dar. Anregungen und Vorschläge sind ausdrücklich willkommen.

\section{Korrespondenzadresse}

\section{Dr. J. Knitza, MHBA}

Medizinische Klinik 3 - Rheumatologie und Immunologie, Universitätsklinikum Erlangen, Friedrich-Alexander-Universität ErlangenNürnberg (FAU)

Ulmenweg 18, 91054 Erlangen, Deutschland johannes.knitza@uk-erlangen.de

\section{Prof. Dr. J. G. Richter}

Poliklinik und Funktionsbereich Rheumatologie \& Hiller-Forschungszentrum Rheumatologie, Medizinische Fakultät, Universitätsklinikum Düsseldorf, Heinrich-Heine-Universität Düsseldorf

Moorenstr. 5, 40225 Düsseldorf, Deutschland jutta.richter@med.uni-duesseldorf.de

Funding. Open Access funding provided by Projekt DEAL.

Interessenkonflikt. J. Knitza, J. Callhoff, G. Chehab, A. Hueber, U. Kiltz, A. Kleyer, M. Krusche, D. Simon, C. Specker, M. Schneider, A. Voormann, M. Welcker und J.G. Richter geben an, dass kein Interessenkonflikt besteht.
Open Access. Dieser Artikel wird unter der Creative Commons Namensnennung 4.0 International Lizenz veröffentlicht, welche die Nutzung, Vervielfältigung, Bearbeitung, Verbreitung und Wiedergabe in jeglichem Medium und Format erlaubt, sofern Sie den/die ursprünglichen Autor(en) und die Quelle ordnungsgemäß nennen, einen Link zur Creative Commons Lizenz beifügen und angeben, ob Änderungen vorgenommen wurden.

Die in diesem Artikel enthaltenen Bilder und sonstiges Drittmaterial unterliegen ebenfalls der genannten Creative Commons Lizenz, sofern sich aus der Abbildungslegende nichts anderes ergibt. Sofern das betreffende Material nicht unter der genannten Creative Commons Lizenz steht und die betreffende Handlung nicht nach gesetzlichen Vorschriften erlaubt ist, ist für die oben aufgeführten Weiterverwendungen des Materials die Einwilligung des jeweiligen Rechteinhabers einzuholen.

Weitere Details zur Lizenz entnehmen Sie bitte der Lizenzinformation auf http://creativecommons.org/ licenses/by/4.0/deed.de.

\section{Literatur}

1. AWMF (2020) Fachgesellschaften. https:// www.awmf.org/fachgesellschaften/ mitgliedsgesellschaften/visitenkarte/fg/ deutsche-gesellschaft-fuer-rheumatologie-evdgrh.html.Zugegriffen:21. Febr. 2020

2. Deutsche Gesellschaft für Rheumatologie e.V. (2020) Therapie-Überwachung. https://dgrh. de/Start/Versorgung/Therapieüberwachung/ Therapieüberwachungsbögen.html. Zugegriffen: 13 . Mai 2020

3. Wilkinson MD, Dumontier M, Aalbersberg IJ et al (2016) The FAIR guiding principles for scientific data management and stewardship. Sci Data 3:160018

4. Knitza J, Vossen D, Geffken I et al (2018) Use of medical apps and online platforms among German rheumatologists: Results of the 2016 and 2018 DGRh conference surveys and research conducted by rheumadocs. ZRheumatol 78(9):839-846

5. Knitza J, TascilarK, MessnerE-Metal (2019) German mobile apps in rheumatology: review and analysis using the mobile application rating scale (MARS). JMIRMhealth Uhealth 7:e14991

6. Stoyanov SR, Hides L, Kavanagh DJ et al (2015) Mobile app rating scale: a new tool for assessing the quality of health mobile apps. JMIR mHealth uHealth 3:e27

7. Najm A, Gossec L, Weill C et al (2019) Mobile health apps for self-management of rheumatic and musculoskeletal diseases: systematic literature review. JMIRMhealth Uhealth 7:e14730

8. Najm A, Nikiphorou E, Kostine Metal (2019) EULAR points to consider for the development, evaluation and implementation of mobile health applications aiding self-management in people living with rheumatic and musculoskeletal diseases. RMD Open 5:e1014

9. Knitza J, Raab C, Lambrecht A et al (2019) The urge for mobile Apps in rheumatology-a German patient perspective [abstract]. Arthritis Rheumatol. 71 (suppl 10). https://acrabstracts.org/abstract/ the-urge-for-mobile-apps-in-rheumatology-agerman-patient-perspective/. Zugegriffen: 13. Mai 2020
10. Richter J, Bungartz C, Weiß A et al (2019) Mobile responsive app-useful additional tool for data collection in the German pregnancy register Rhekiss? Arthritis Rheumatol 71 (suppl 10). https://acrabstracts.org/abstract/mobileresponsive-app-useful-additional-tool-for-datacollection-in-the-german-pregnancy-registerrhekiss/.Zugegriffen: 13. Mai 2020

11. Stack RJ, Nightingale P, Jinks C et al (2019) Delays between the onset of symptoms and first rheumatology consultation in patients with rheumatoid arthritis in the UK: an observational study. BMJOpen 9:e24361

12. Raza K, Stack R, Kumar K et al (2011) Delays in assessment of patients with rheumatoid arthritis: variations across Europe. Ann Rheum Dis 70:1822-1825

13. Quinn MA, Emery P (2003) Window of opportunity in early rheumatoid arthritis: possibility of altering the disease process with early intervention. Clin Exp Rheumatol 21:154-157

14. Benesova K, LorenzHM, Lion Vetal (2019) Früh-und Screeningsprechstunden: Ein notwendiger Weg zur besseren Frühversorgung in der internistischen Rheumatologie? ZRheumatol 78:722-742

15. Powley L, Mcilroy G, Simons G et al (2016) Are online symptoms checkers useful for patients with inflammatory arthritis? BMC Musculoskelet Disord 17:362

16. Richter JG, Becker A, Koch Tet al (2009) Internet use in rheumatology outpatients in 2006: gender less important. Clin Exp Rheumatol 27:15-21

17. Feuchtenberger $M$, Nigg AP, Kraus $M R$ et al (2016) Rate of proven rheumatic diseases in a large collective of referrals to an outpatient rheumatology clinic under routine conditions. Clin Med Insights Arthritis Musculoskelet Disord 9:181-187

18. Holle JU, Moosig F (2019) Drei Jahre Terminservicestelle: hohe Rate an Fehlzuweisungen und Noshow-Patienten - Eine retrospektive Auswertung des Rheumazentrum Schleswig-Holstein Mitte. ZRheumatol 78:832-838

19. Knitza J, Knevel R, Raza K et al (2020) Toward earlier diagnosis using combined ehealth tools in rheumatology: the joint Pain Assessment Scoring Tool (JPAST) project. JMIR Mhealth Uhealth. https://doi.org/10.2196/17507

20. Schwarting A, Dreher M, Assmann G et al (2019) Erfahrungen und Ergebnisse aus Rheuma-VOR. ZRheumatol 78:743-752

21. Proft F, Spiller L, Protopopov M et al (2019) Development of an optimized online self-referral tool for early recognition of patientins axial spondyloarthritis (AXSPA) —-Data from the 'OPTIREF'Study. Ann Rheum Dis 78:486

22. Richter JG, Wessel E, Klimt R et al (2008) RheumaCheck: Entwicklung und Evaluation eines deutschsprachigen Rheuma-Screening Instruments. Wien Klin Wochenschr 120:103-111

23. Urruticoechea-Arana A, León-Vázquez F, GinerRuiz V et al (2019) Development of an application for mobile phones (App) based on the collaboration between the Spanish Society of Rheumatology and Spanish Society of Family Medicine for the referral of systemic autoimmune diseases from primary care to rheumatology. Rheumatol Clin. https://doi.org/10.1016/j.reuma. 2019.09.001

24. Kampling C, Chehab G, Schneider M et al (2014) Selbst-Monitoring bei entzündlich-rheumatischen Erkrankungen. ZRheumatol 73:706-713

25. Ragouzeos D, Gandrup J, Berrean B et al (2019) "Am I OK?" using human centered design to 
empower rheumatoid arthritis patients through patient reported outcomes. Patient Educ Couns 102:503-510

26. Fautrel B, Alten R, Kirkham B et al (2018) Call for action: how to improve use of patient-reported outcomes to guide clinical decision making in rheumatoid arthritis. Rheumatol Int 38:935-947

27. Richter JG, Chehab G, Schneider M (2016) Electronic health records in rheumatology: emphasis on automated scoring and additional use. Clin Exp Rheumatol 34:S62-S68

28. Richter J, Chehab G, Tomczak Met al (2019) PICASO - Die Plattform für eine verbesserte persönliche und koordinierte Betreuung chronisch Kranker Erste Zwischenergebnisse einer Proof-of-Concept Studie. German Medical Science GMS, Düsseldorf https://doi.org/10.3205/19dgrh09547

29. Nannen C, Chehab G, Acar H et al (2018) Frequent use of app diary functions in a digital health project with rheumatoid arthritis patients. German Medical Science GMS, Düsseldorf https://doi.org/ 10.3205/18dgrh061

30. Bingham $\mathrm{CO}$, Gaich $\mathrm{CL}$, Delozier $\mathrm{AM}$ et al (2019) Use of daily electronic patient-reported outcome (PRO) diaries in randomized controlled trials for rheumatoid arthritis: rationale and implementation. Trials 20:182

31. Krusche M, Klemm P, Grahammer M et al (2020) Electronic patient-reported outcomes: a survey about acceptance, usage and barriers among German rheumatologists. JMIR Mhealth Uhealth. https://doi.org/10.2196/18117

32. El Miedany Y, El Gaafary M, El Aroussy N et al (2017) Toward electronic health recording: evaluation of electronic patient reported outcome measures (e-PROMs) system for remote monitoring of early systemic lupus patients. Clin Rheumatol 36:2461-2469

33. Riemekasten G, Aringer M, Baerwald CGO et al (2016) Rheumatologie - Integration in die studentische Ausbildung (RISA). Z Rheumatol 75:493-501

34. Kleyer A, Simon D, Hartmann F et al (2019) "Virtual rheumatology": A new teaching concept for rheumatology of the future? Z Rheumatol 78:112-115

35. Knitza J, Kleyer A, Klüppel M et al (2020) OnlineUltraschallernmodule in der Rheumatologie. ZRheumatol 79:276-279

36. BfArM (2020) Digitale Gesundheitsanwendungen. https://www.bfarm.de/DE/Medizinprodukte/ DVG/ node.html.Zugegriffen: 22. Febr. 2020

37. https://www.bgbl.de/xaver/bgbl/start.xav? startbk=Bundesanzeiger_BGBI\& jumpTo=bgbl120s0768.pdf-_ bgbl__\%2F\%2F* \%5B\%40attr_id\%3D\%27bgbl120s0768.pdf\%27 \%5D_1589046217953.Zugegriffen: 13. Mai 2020

38. Bundesministerium für Gesundheit (2020) Stellungnahmen zum Referentenentwurf Digitale-Versorgung-Gesetz. https://www. bundesgesundheitsministerium.de/service/ gesetze-und-verordnungen/guv-19-Ip/ stellungnahmen-refe/dvg.html. Zugegriffen: 9. März 2020

\section{Hohes Thrombose-Risiko bei Menschen mit rheumatischen und muskuloskelettalen Erkrankungen}

Weniger venöse Thrombosen durch TNF-Hemmer

Patienten mit rheumatoider Arthritis (RA) und hoher Krankheitsaktivität leiden besonders häufig unter Thrombosen. Bei Patienten mit hoher Krankheitsaktivität entwicklet einer von hundert innerhalb eines Jahres eine venöse Thromboembolie - das entspricht einem Anstieg um mehr als das Zweifache im Vergleich zu Patienten in Remission (1). Eine Therapie mit biological disease-modifying antirheumatic drugs (bDMARD) kann das erhöhte Thrombose-Risiko reduzieren, zeigen Daten des deutschen RABBIT1-Registers (2).

Aufgrund chronischer Entzündungen, unter denen Patienten mit RA leiden, erhöht sich für sie das Risiko gefährlicher Beinvenen- und Lungenthrombosen um das Zwei- bis Dreifache (3). Welche Faktoren das Entstehen einer Thrombose hier begünstigen und welche Medikamente das Risiko reduzieren könnten, wurde jetzt in zwei aktuellen Studien untersucht:

\section{1 von 100 RA-Patienten mit hoher Krankheitsaktivität erleidet eine Thrombose}

Eine schwedische Kohorten-Studie ging der Frage nach, ob der Grad der Krankheitsaktivität das Thrombose-Risiko beeinflusst (1). Das Team um V. Molander, Karolinska Institutet Stockholm, hat die Daten von 46311 Patienten mit RA aus dem schwedischen Qualitätsregister für Rheumatologie (SRQ) über einen Zeitraum von zwölf Jahren analysiert.

Die Studie belegt einen starken Zusammenhang zwischen der mit dem DAS28 gemessenen Krankheitsaktivität der RA und dem Risiko einer VTE: „Unter den Patienten mit hohen Entzündungswerten wird einer von hundert innerhalb des kommenden Jahres eine VTE entwickeln - das entspricht einer mehr als zweifachen Zunahme im Vergleich zu Patienten in Remission", fasst Molander die Ergebnisse zusammen.

\section{Biologika können Thromboserisiko reduzieren}

Ob der Einsatz von bDMARDs wie TNF-Inhibitoren im Vergleich zu csDMARDs das Thrombose-Risiko reduziert, haben Wissenschaftler um den Erstautor Dr. M. Schäfer vom Programmbereich Epidemiologie des Deutschen Rheuma-Forschungszentrums in Berlin untersucht. Hierfür hat das Team die Daten von mehr als 11000 RA-Patientinnen und -Patienten aus dem deutschen RABBIT ${ }^{1}$ -
Register analysiert, die nach mindestens einem csDMARD-Versagen entweder mit einem weiteren csDMARD behandelt oder auf die Therapie mit einem bDMARD umgestellt wurden.

„Die Behandlung mit TNF-Hemmern reduzierte das Risiko schwerer VTE-Ereignisse im Vergleich zu csDMARDs um fast die Hälfte", erklärt Schäfer. Eine erhöhte Entzündungsaktivität war auch anhand der RABBIT-Daten mit einem signifikant erhöhten Risiko für VTEs assoziiert: Ein CRP-Wert von mindestens $5 \mathrm{mg} / \mathrm{l}$ verdoppelte das Risiko annähernd. „Bei Patienten mit erhöhtem ThromboseRisiko sollte daher eine alternative Therapie mit TNF-Hemmern statt der Standardbehandlung mit csDMARD in Erwägung gezogen werden", resümiert PD Dr. A. Strangfeld, Studienleiterin des RABBIT-Registers in Berlin und Mitautorin der Studie. „Darüber hinaus ist die Senkung der Entzündungsaktivität ein wichtiger Faktor, um das Risiko für VTEs zu vermindern."

Literatur

1.) Molander V, Bower $H$, Askling J. Does the risk of venous thromboembolism vary with disease activity in rheumatoid arthritis? DOI: 10.1136/annrheumdis-2020-eular.353

2.) Schäfer $M$, Schneider $M$, Graessler $A$ et al. TNF inhibitors are associated with a reduced risk of venous thromboembolism compared to csDMARDs in RA patients DOI: 10.1136/annrheumdis-2020-eular.1505

3.) Chung W, Peng C, Lin C et al. Rheumatoid arthritis increases the risk of deep vein thrombosis and pulmonary thromboembolism: a nationwide cohort study. Annals of the Rheumatic Diseases 2014;73:1774-1780. 